\title{
How to Determine Fermi Vectors by Angle-Resolved Photoemission
}

\author{
L. Kipp, ${ }^{1}$ K. Roßnagel, ${ }^{1}$ C. Solterbeck, ${ }^{2}$ T. Strasser, ${ }^{2}$ W. Schattke, ${ }^{2}$ and M. Skibowski ${ }^{1}$ \\ ${ }^{1}$ Institut für Experimentelle und Angewandte Physik, Universität Kiel, D-24098 Kiel, Germany \\ ${ }^{2}$ Institut für Theoretische Physik und Astrophysik, Universität Kiel, D-24098 Kiel, Germany
}

(Received 27 May 1999)

\begin{abstract}
Angle-resolved photoemission spectroscopy (ARPES) has been commonly applied to evaluate the shape of Fermi surfaces by employing simple criteria, such as maximum photoemission intensity at the Fermi level or discontinuity in the momentum distribution function, for the determination of the Fermi vector $k_{F}$ parallel to the surface. Here we show that these criteria may lead to large uncertainties, in particular for narrow band systems. We develop a reliable method for the determination of Fermi vectors employing high resolution ARPES at different temperatures. The relevance and accuracy of the method is discussed on data of the quasi-two-dimensional system $\mathrm{TiTe}_{2}$.
\end{abstract}

PACS numbers: 71.18.+y, 79.60.-i

A wide variety of physical phenomena of crystalline materials, such as, e.g., transport, optical and magnetic response, and phase transitions, rely on details of the topology of the Fermi surface (FS). Its experimental determination, performed by traditional techniques such as the de Haas-van Alphen effect, magnetoacoustic effect, Compton scattering, or positron annihilation, has, however, been restricted to bulk materials. All techniques have in common that they provide indirect information on the shape of the Fermi surface. While the first two determine extremal cross sections of FS's in a plane normal to the applied magnetic field, the latter yield information on oneand two-dimensional projections of FS's. More complex cases, such as superlattices, heterostructures, or even clean surfaces, can also hardly be accessed. Angle-resolved photoemission spectroscopy (ARPES) has emerged as probably the most powerful tool for determining the occupied electronic band structure of solids and their surfaces. Recently, it has been extensively applied to gain insight into the topology of Fermi surfaces of a variety of materials ranging from conventional three-dimensional metals such as $\mathrm{W}$ and $\mathrm{Cu}[1-3]$ to quasi-two-dimensional layered compounds [4-6], purple bronzes [7], and high $T_{c}$ cuprate materials $[8,9]$. The accuracy of the determination of the Fermi surface by ARPES, however, has never been questioned and it turns out that even for the extensively studied $\mathrm{BiSrCaCuO}$ it is not at all clear that the topology of the normal state FS shows holelike pockets around the corners of the Brillouin zone (BZ) [8-10] or electron pockets around the center of the BZ [11].

It is widely assumed that ARPES measures the spectral function $A(\mathbf{k}, \omega)$ of the one-particle system times the Fermi function $f(\omega)$, and matrix elements do not play a significant role (see, e.g., Refs. [12,13]). This motivates studies of band dispersions, line shapes, momentum distribution functions, and Fermi surfaces. Fermi vectors have been extracted from ARPES data employing criteria such as (i) maximum ARPES intensity at the Fermi level $E_{F}$ $[1,2,7,8,14]$, (ii) $\max \left|\nabla_{k}\right|$ of the energy integrated photoemission intensity $[4,5,15,16]$, or (iii) fitting ARPES peak positions over several emission angles and extrapolating the dispersion to $E_{F}$ [17]. However, none of these techniques explicitly considers the detailed mechanism of the photoemission process. In particular, matrix element effects and differences between photocurrent and spectral function have been totally neglected and make these simple interpretations highly questionable. Although, in most cases, photoemission peak positions still resemble locations of electronic bands, the shape of spectral functions is in general not directly reproduced by the photocurrent $[18,19]$. If photoemission calculations within the onestep model are not available, reliable simple procedures for an analysis of high resolution photoemission data are needed.

Employing high resolution photoemission spectroscopy, we show in this Letter how Fermi vectors can be determined with high accuracy when intensity modifications due to the photoemission process are explicitly eliminated by comparing photoemission spectra taken at different temperatures. The reliability and applicability of the method will be demonstrated on the layered Fermi liquid reference material $1 T-\mathrm{TiTe}_{2}$. It does not show an indication of charge density waves and exhibits $\mathrm{Ti} 3 d$ conduction band emissions which are well separated from other bands [20-22]. It may thus serve as a model system for assessing the accuracy of the determination of the Fermi surface of quasi-two-dimensional systems.

All results described here were obtained on clean chemical vapor transport grown $1 T-\mathrm{TiTe}_{2}$ samples prepared by cleavage in ultrahigh vacuum. Photoemission spectra were taken with synchrotron radiation supplied from the storage ring DORIS III at Hamburg Synchrotron Radiation Laboratory using our angular spectrometer for photoelectrons with high energy resolution. The electrons were detected by use of a $180^{\circ}$ spherical analyzer mounted on a two-axes goniometer with an absolute angular precision of better than $0.1^{\circ}$. The overall energy resolution was chosen to $30 \mathrm{meV}$. The position of the Fermi level was determined from spectra of polycrystalline gold with an accuracy of $\pm 1 \mathrm{meV}$. 
The photocurrent within the sudden approximation is given by the spectral representation of the one-particle Green function: $I(\mathbf{k}, \omega)=I_{0}(\mathbf{k}) f(\omega) A(\mathbf{k}, \omega)$, where $f$ is the Fermi function and $A(\mathbf{k}, \omega)$ is the one-particle spectral function. The prefactor $I_{0}$ involves the transition matrix element and is thus $\mathbf{k}$ dependent. As will be shown below, this $\mathbf{k}$ dependence crucially affects the reliability of the determination of $\mathbf{k}_{F}$. Depending on photon energy, the component of $\mathbf{k}$ perpendicular to the surface $k_{\perp}$ is varied in angle-resolved photoemission. It further complicates a full three-dimensional determination of the Fermi vector. This, however, is going beyond the scope of this Letter and will be discussed in a forthcoming publication.

The component of the Fermi vector parallel to the surface $\mathbf{k}_{F \|}$ can be identified by locating the peak of $A(\mathbf{k}, \omega)$ at zero binding energy. This is not a trivial matter since the photocurrent corresponds to $f(\omega) A(\mathbf{k}, \omega)$, and the peak in the photocurrent is what results when the Fermi function cuts off the peak of the spectral function $A(\mathbf{k}, \omega)$. Different approaches have been used to solve this difficulty: (i) find the peak in $I\left(\mathbf{k}, E_{F}\right)$ (maximum intensity at the Fermi level); (ii) use the sum rule [23] relating the energy integrated spectral function to the momentum distribution function: $n(\mathbf{k})=\int_{-\infty}^{\infty} d \omega f(\omega) A(\mathbf{k}, \omega)$. By definition, the momentum vectors $\mathbf{k}_{F}$ constituting the Fermi surface are identified through a step or at least the vertical slope of the momentum electron distribution $n(\mathbf{k})$ at zero temperature $[4,15]$. Method (iii) employs a fit of photoemission peak positions over a wide range of emission angles and extrapolation to zero binding energy to obtain an estimate for $\mathbf{k}_{F \|}$.

In Figs. 1(a) and 1(b) we show the results of a simulation for a narrow band represented by a Gaussian peak (width: $\Delta E_{\mathrm{FWHM}}=100 \mathrm{meV}$, dispersion: $\Delta E / \Delta k=-0.2 \mathrm{eV} \AA$ ) crossing the Fermi level at $k_{\|}=0 \AA^{-1}$. Fermi vectors determined using the approaches (i) and (ii) are plotted as a function of the experimental resolution. Solid lines show results for a matrix element $I_{0}(k)=$ const, shaded areas for a weakly changing $I_{0}(k) \sim a(k+1),-1 \leq a \leq+1$. Both approaches provide values for $k_{F \|}$ revealing systematic deviations of up to about $20 \%$ (with respect to the distance $\Gamma M=0.96 \AA^{-1}$ of the Brillouin zone) strongly depending on the experimental resolution. It should be noted here that these approaches may be additionally affected in strongly correlated Fermi liquid systems where incoherent backgrounds (e.g., electron hole pairs) dominate the modulation of $n(\mathbf{k})$ [24].

A reliable approach for the determination of $\mathbf{k}_{F \|}$ is to compare spectra at different temperatures ( $\Delta T$ method). Centered at $E_{F}$ and $\mathbf{k}_{F}$ and with respect to $\omega$, the difference of the Fermi functions for temperatures $T_{1}$ and $T_{2}$ is an odd function, $A\left(\mathbf{k}_{F}, \omega\right)$ is even, and therefore the integral of their product over a symmetric energy window vanishes. In practice, the integration is replaced by the finite energy resolution given by a symmetric analyzer function
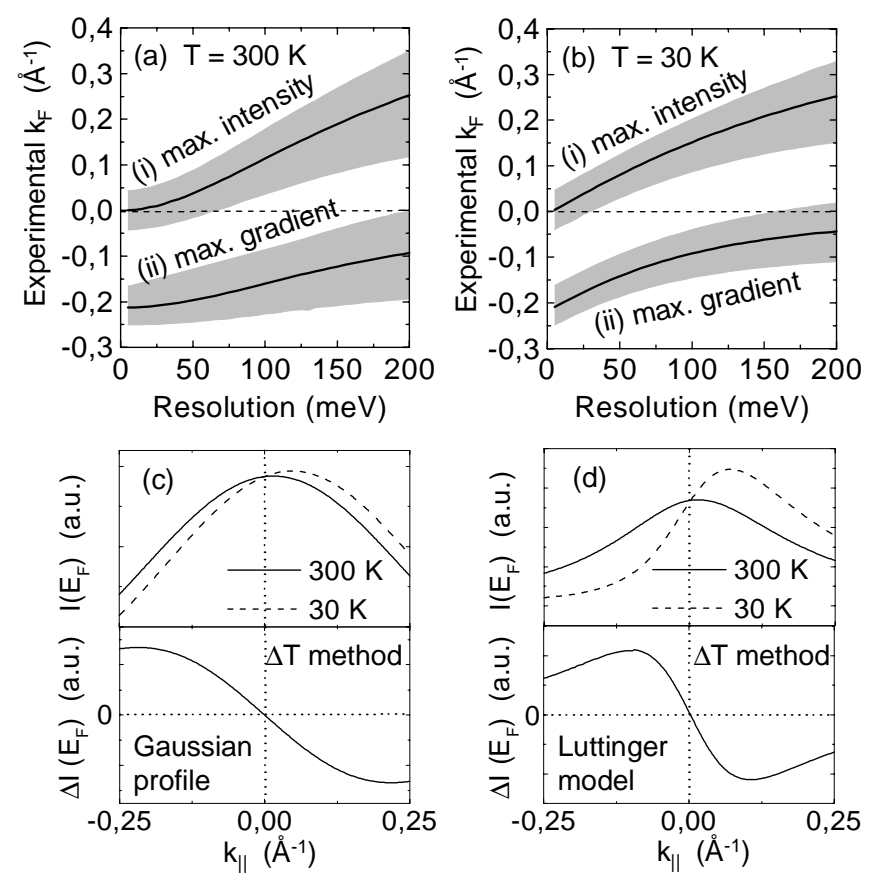

FIG. 1. Systematic deviations for $k_{F \|}$ determined by experimental procedures employing methods (i) $\max \left[I\left(\mathbf{k}, E_{F}\right)\right]$ and (ii) $\max \left[\left|\nabla_{k} n(k)\right|\right]$ plotted as a function of experimental resolution (Gaussian type) for $300 \mathrm{~K}$ (a) and $30 \mathrm{~K}$ (b). Simulation for a Gaussian peak (width: $\Delta E_{\mathrm{FWHM}}=100 \mathrm{meV}$, dispersion: $\Delta E / \Delta k=-0.2 \mathrm{eV} \AA$ ) crossing the Fermi level at $k_{F \|}=0 \AA^{-1}$. Solid lines and shaded areas represent simulations for constant and varying matrix elements, respectively. Simulation results for the $\Delta T$ method are shown in (c) and (d) (resolution $30 \mathrm{meV}$ ). See text for details.

$w$, which vanishes outside $[-\epsilon, \epsilon]$. The difference of the intensities taken at $E_{F}=0$ is

$$
\begin{aligned}
\Delta I\left(\mathbf{k}_{\|}\right)= & I_{0}\left(\mathbf{k}_{\|}\right) \int_{-\epsilon}^{\epsilon} d \omega A(\mathbf{k}, \omega) \\
& \times\left[f_{T_{1}}(\omega)-f_{T_{2}}(\omega)\right] w(\omega),
\end{aligned}
$$

assuming that for the intervals of interest $A$ is independent of temperature and $I_{0}$ of temperature and energy. It then follows from $\Delta I\left(\mathbf{k}_{\|}\right)=0$ that $\mathbf{k}_{\|}=\mathbf{k}_{F \|}$. This is valid for all $A$ which satisfy in $[-\epsilon, \epsilon]$ that $A\left(\mathbf{k}_{F}, \omega\right)=A\left(\mathbf{k}_{F},-\omega\right)$ and $A(\mathbf{k}, \omega) \neq A(\mathbf{k},-\omega)$ for $\mathbf{k} \neq \mathbf{k}_{F}$. Then at general $\mathbf{k}$ the differences of $A$ do not change sign and

$$
\begin{aligned}
\Delta I= & I_{0} \int_{-\epsilon}^{0} d \omega[A(\mathbf{k}, \omega)-A(\mathbf{k},-\omega)] \\
& \times\left[f_{T_{1}}-f_{T_{2}}\right] w \neq 0 .
\end{aligned}
$$

A variety of spectral functions fulfill these conditions, including Lorentzians, Gaussians, Voigt profiles, the Luttinger model [25], a suggestion by Matho [21,26,27], two-dimensional [28], and marginal [29] Fermi liquids. A high energy resolution extends the scope. The independence of temperature of such integrals in the vicinity of $\mathbf{k}_{F}$ and $E_{F}$ has already been tested [23] and will later be shown again for the present case. With a high $\mathbf{k}_{\|}$ 
resolution, the criterion is still valid since for a symmetric band dispersion [with respect to $\left(E_{F}, \mathbf{k}_{F}\right)$ ] the additional contributions annihilate. The accuracy might be lowered due to $\mathbf{k}$ dependencies of $I_{0}$. This possible influence, however, can directly be excluded when the experimental $k_{\|}$resolution windows lies well within a regime where point symmetry of $\Delta I\left(k_{\|}\right)$around $\Delta I=0$ is observed in the experimental data. Note that for a tiny energy interval the required fixed-k mode of photoemission coincides with the employed fixed-angle mode well within the angular resolution. A simulation of the $\Delta T$ method for a Gaussian profile and the Luttinger model is shown in Figs. 1(c) and 1(d). The Fermi vector $\mathbf{k}_{\|}=0$ is exactly determined by $\Delta I=0$ in both cases for all resolutions.

In Fig. 2(a) we show high energy and angle-resolved photoemission spectra of $1 T$-TiTe $_{2}$ along the $\Gamma M$ direction of the Brillouin zone. For the Ti $3 d$ band which is well separated from contributions of other bands, one observes besides the crossing of the band connected with an intensity break down a characteristic behavior of the line shape. Line shape studies have been performed employing either a Fermi liquid scenario using a many body spectral function with an imaginary part of the self-energy depending quadratically on energy referred to $E_{F}$ [20]or profiles suggested by Matho [21,27] for approximating the spectral function $A(\mathbf{k}, \omega)$. In the close vicinity of the Fermi vector the experimental spectra could excellently be fitted. But for emission angles larger than $\sim 18^{\circ}$ the quality of the fits is drastically reduced. Values obtained for the Fermi vector were varying between $14^{\circ}\left(0.51 \AA^{-1}\right)$ [21] and $14.75^{\circ}$ $\left(0.53 \AA^{-1}\right)[20,22,27]$. These studies, however, did not consider the photoemission process explicitly and intrinsic spectral information can be distorted by varying matrix ele-
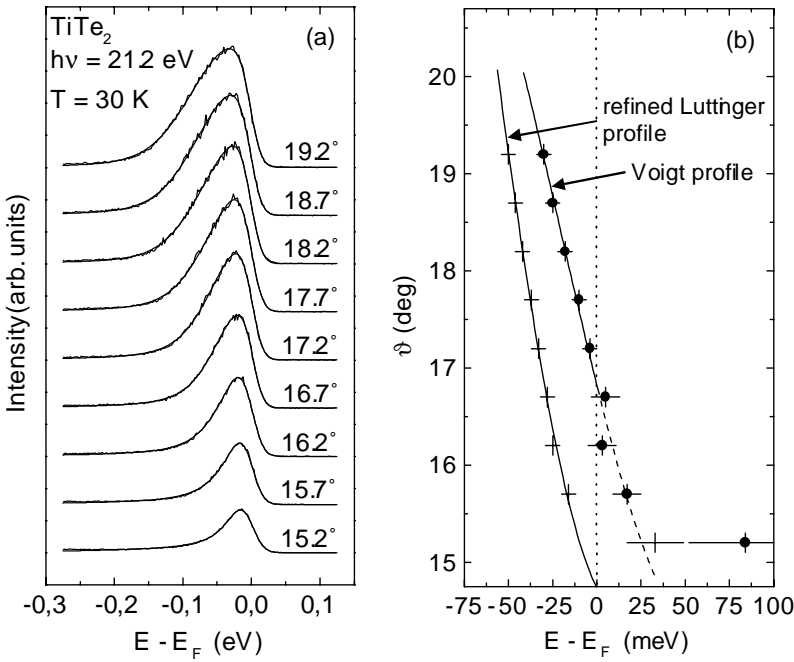

FIG. 2. (a) Angle-resolved photoemission spectra ( $h \nu=$ $21.2 \mathrm{eV}$ ) associated with the $\mathrm{Ti} 3 d$ band of $1 T-\mathrm{TiTe}_{2}$ in the direction $\Gamma M$ taken with high resolution and line shape fit using Voigt profiles. (b) Band dispersion obtained from the fitted peak positions [method (iii)] using Voigt and refined Luttinger profiles. Note the different band crossings at $E_{F}$. ments or other secondary effects. This problem is further illustrated in Fig. 2(a) where an excellent fit (solid lines) can already be achieved for all emission angles employing simple Voigt profiles times the Fermi function convoluted by the spectrometer response. This demonstrates that without explicit consideration of the photoemission process sophisticated spectral functions can hardly be delineated. Peak positions resulting from the fit give the experimental dispersion shown in Fig. 2(b). The band crossing is observed at $16.7^{\circ}$ corresponding to $0.60 \AA^{-1}$. It should be noted here that, in addition to an increased experimental and calculational (fit) effort, the values for $\mathbf{k}_{F \|}$ obtained by method (iii) rely on the profiles employed. For the refined Luttinger profile the band crossing is observed at $14.75^{\circ}$ [see Fig. 2(b)].

For an application of the $\Delta T$ method we plot normalized photoemission spectra of $1 T-\mathrm{TiTe}_{2}$ taken at 100 and $190 \mathrm{~K}$ [Fig. 3(a)]. The broadening of the Fermi function according to temperature is evident. Intensities at the Fermi level $(\Delta E=30 \mathrm{meV})$ and intensities integrated over the whole spectrum are depicted in 3(b) and 3(c), respectively. According to the $\Delta T$ method, the Fermi vector is given by the intersection of the curves in 3(b) which can clearly be identified at a value of $16.6^{\circ} \pm 0.1^{\circ}\left(0.598 \pm 0.004 \AA^{-1}\right)$. For comparison we also show the values for $k_{F}$ obtained using the maximum intensity (i) and maximum gradient (ii) methods described above. The maximum intensity at $E_{F}$ can be observed on a relatively broad peak at around $17.8^{\circ}$ $\left(0.64 \AA^{-1}\right)$ showing the systematic erroneous shift of $k_{F \|}$ towards occupied states as already observed in the simulation of Fig. 1. The energy integrated intensity and its derivative are depicted in Fig. 3(c). Since the gradient of the integrated intensity is only marginally changing in the $k_{\|}$regime of interest, a rather broad maximum is observed making a detailed quantitative comparison with FS calculations very difficult. Temperature effects such as broadening of the quasiparticle peaks [30] may eventually occur [see, e.g., Fig. 3(a), spectra at $19.2^{\circ}$ ]. However, at $k_{F}$ this possible influence can be checked employing symmetrized spectra $I\left(\mathbf{k}_{F}, \omega\right)+I\left(\mathbf{k}_{F},-\omega\right)=I_{0}\left(\mathbf{k}_{F}\right) A\left(\mathbf{k}_{F}, \omega\right)$ eliminating the Fermi function [12,31] which are shown in Fig. 3(d). For the spectra taken at 100 and $190 \mathrm{~K}$ no differences can be observed ruling out any $T$ dependence of $I_{0}\left(\mathbf{k}_{F}\right) A\left(\mathbf{k}_{F}, \omega\right)$ in this temperature range.

In summary, we have developed a reliable and simple method to determine Fermi vectors by angle-resolved photoemission spectroscopy. Employing temperature difference spectra, the photoemission process hiding the spectral function in measured spectra is explicitly considered. Therefore, the method even works for systems showing rapidly changing matrix elements with variation of $\mathbf{k}$. For the layered material $1 T-\mathrm{TiTe}_{2}$ we have demonstrated that the accuracy of the determined $k_{F}$ values can be significantly improved to better than $\pm 0.4 \%$ of the dimension of the Brillouin zone. Compared to other methods revealing up to 1 order of magnitude larger error 

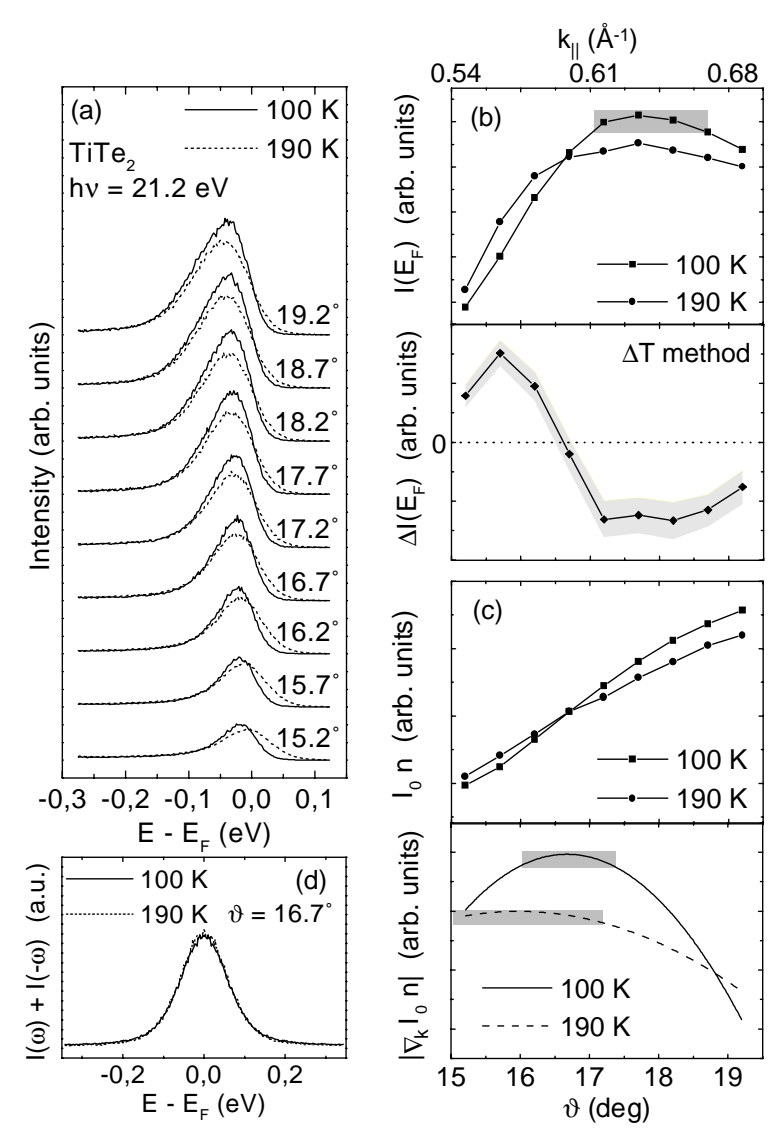

FIG. 3. (a) Angle-resolved photoemission spectra $(\Delta E=30 \mathrm{meV})$ associated with the Ti $3 d$ band of $1 T-\mathrm{TiTe}_{2}$ in the direction $\Gamma M$ taken at 100 and $190 \mathrm{~K}$. (b) Intensities at the Fermi level [method (i)]. The intersection marks the Fermi vector $\left(\Delta T\right.$ method). An uncertainty of $\pm 0.1^{\circ}$ (hatched area) emerges from the error bar of the Fermi energy $( \pm 1 \mathrm{meV})$. (c) Intensities integrated over the whole spectrum and $k$ derivative (smoothed) of the integrated intensity [method (ii)]. Grey bars mark the uncertainties for the determination of $k_{F}(95 \%$ of the maximum value). (d) Symmetrized spectra showing no differences for spectral functions at 100 and $190 \mathrm{~K}$.

bars together with systematic deviations of up to $20 \%$ reliable quantitative comparisons between experiment and Fermi surface calculations going beyond similarities of the shapes will now become possible.

Experimental support by J. Brügmann, R. Schwedhelm, and S. Woedtke is gratefully acknowledged.
This research is supported by the BMBF, FR Germany (Projects No. 05 SB8 FKB, No. 05 SE8 FKA, and No. 05 SB8 FKA).

[1] E. Rotenberg and S. D. Kevan, Phys. Rev. Lett. 80, 2905 (1998).

[2] J. Avila et al., J. Vac. Sci. Technol. A 13, 1501 (1995).

[3] P. Aebi et al., Surf. Sci. 307, 917 (1994).

[4] T. Straub et al., Phys. Rev. B 55, 13473 (1997).

[5] R. Claessen, J. Electron Spectrosc. Relat. Phenom. 92, 53 (1998).

[6] M. Boehme, L. Kipp, and M. Skibowski, Solid State Commun. 112, 101 (1999).

[7] G. Gweon et al., Phys. Rev. B 55, R13 353 (1997).

[8] P. Aebi et al., Phys. Rev. Lett. 72, 2757 (1994).

[9] N. L. Saini et al., Phys. Rev. Lett. 79, 3467 (1997).

[10] H. Ding et al., Phys. Rev. Lett. 76, 1533 (1996).

[11] Y. Chuang et al., Phys. Rev. Lett. 83, 3717 (1999).

[12] M. R. Norman et al., Nature (London) 392, 157 (1998).

[13] M. R. Norman, M. Randeria, H. Ding, and J.C. Campuzano, Phys. Rev. B 57, R11 093 (1998).

[14] A. Santoni, L. J. Terminello, F. J. Himpsel, and T. Takahashi, Appl. Phys. A 52, 229 (1991).

[15] J. Campuzano et al., Phys. Rev. B 53, R14 737 (1996).

[16] F. Ronning et al., Science 282, 2067 (1998).

[17] Z.X. Shen and D. S. Dessau, Phys. Rep. 253, 1 (1995).

[18] A. Bödicker and W. Schattke, J. Electron Spectrosc. Relat. Phenom. 76, 265 (1995).

[19] C. Solterbeck et al., Phys. Rev. Lett. 79, 4681 (1997).

[20] R. Claessen et al., Phys. Rev. Lett. 69, 808 (1992).

[21] S. Harm et al., J. Electron Spectrosc. Relat. Phenom. 68, 111 (1994).

[22] R. Claessen et al., Phys. Rev. B 54, 2453 (1996).

[23] M. Randeria et al., Phys. Rev. Lett. 74, 4951 (1995).

[24] K. Matho, Physica (Amsterdam) 199B-200B, 382 (1994).

[25] J. M. Luttinger, Phys. Rev. 121, 942 (1961).

[26] K. Matho, J. Phys. Chem. Solids 156, 1735 (1995).

[27] J. Allen, G. Gweon, R. Claessen, and K. Matho, J. Phys. Chem. Solids 56, 1849 (1995).

[28] C. Hodges, H. Smith, and J. W. Wilkins, Phys. Rev. B 4, 302 (1971).

[29] C. M. Varma et al., Phys. Rev. Lett. 63, 1996 (1989).

[30] J. J. Joyce et al., Phys. Rev. B 54, 17515 (1996).

[31] Assuming $A\left(\mathbf{k}_{\mathbf{F}}, \omega\right)=A\left(\mathbf{k}_{\mathbf{F}},-\omega\right)$. 